\title{
Osvaldo and Isis retrotransposons as markers of the Drosophila buzzatii colonisation in Australia
}

\author{
María Pilar García Guerreiro ${ }^{*}$ and Antonio Fontdevila
}

\begin{abstract}
Background: Transposable elements (TEs) constitute an important source of genetic variability owing to their jumping and regulatory properties, and are considered to drive species evolution. Several factors that are able to induce TE transposition in genomes have been documented (for example environmental stress and inter- and intra-specific crosses) but in many instances the reasons for TE mobilisation have yet to be elucidated. Colonising populations constitute an ideal model for studying TE behaviour and distribution as they are exposed to different environmental and new demographic conditions. In this study, the distribution of two TEs, Osvaldo and Isis, was examined in two colonising populations of D. buzzatii from Australia. Comparing Osvaldo copy numbers between Australian and Old World (reported in previous studies) colonisations provides a valuable tool for elucidating the colonisation process and the effect of new conditions encountered by colonisers on TEs.

Results: The chromosomal distributions of Osvaldo and Isis retrotransposons in two colonising populations of D. buzzatii from Australia revealed sites of high insertion frequency (>10\%) and low frequency sites. Comparisons between Osvaldo insertion profiles in colonising populations from the Old World and Australia demonstrate a tendency towards a higher number of highly occupied sites with higher insertion frequency in the Old World than in Australian populations. Tests concerning selection against deleterious TE insertions indicate that Isis is more controlled by purifying selection than Osvaldo. The distribution of both elements on chromosomal arms follows a Poisson distribution and there are non-significant positive correlations between highly occupied sites and chromosomal inversions.

Conclusions: The occupancy profile of Osvaldo and Isis retrotransposons is characterised by the existence of high and low insertion frequency sites in the populations. These results demonstrate that Australian D. buzzatii populations were subjected to a founder effect during the colonisation process. Moreover, there are more sites with high insertion frequency in the Old World colonisation than in the Australian colonisation, indicating a probable stronger bottleneck effect in Australia. The results suggest that selection does not seem to play a major role, compared to demography, in the distribution of transposable elements in the Australian populations.
\end{abstract}

\section{Background}

Transposable elements (TEs) are DNA sequences that can move along the genome and constitute an important fraction of the genomes of most organisms studied so far [1]. It is indisputable that genomes have evolved in close association with TEs, which are characterised by high species specificity and high structural variability, even in closely related organisms. After several decades of experimental and theoretical work, the mechanisms controlling TE copy

\footnotetext{
* Correspondence: mariapilar.garcia.guerreiro@uab.es
Grup de Biología Evolutiva. Departament de Genètica i Microbiologia,

* Correspondence: mariapilar.garcia.guerreiro@uab.es
Grup de Biología Evolutiva. Departament de Genètica i Microbiologia,

Facultat de Biociències, Universitat Autònoma de Barcelona, 08193 Bellaterra (Barcelona), Spain
}

(c) 2011 García Guerreiro and Fontdevila; licensee BioMed Central Ltd. This is an Open Access article distributed under the terms of the Creative Commons Attribution License (http://creativecommons.org/licenses/by/2.0), which permits unrestricted use, distribution, and reproduction in any medium, provided the original work is properly cited.

numbers in populations and most aspects of their biology within species are still not well understood. Classical theoretical models predict that autonomous elements can be present in the genomes of a population as long as their steady transposition rates can counterbalance their elimination by natural selection $[2,3]$. This equilibrium hypothesis was considered the principal explanation for the maintenance of a constant TE copy number. However, not all populations are at equilibrium. For instance: first, episodic transposition bursts promote TE genome invasion in natural populations exposed to different population and environmental regimes; second, in other population conditions such as weak selection [4] or migration rates lower 
than transposition rates [5], it takes a substantial time period to reach equilibrium; third, stress environmental conditions and other population phenomena, including demographic (as in our case) and mating processes, are among the prime causes for the distribution of TEs in genomes. Data concerning plants [6] and nematodes [7] demonstrate that demographic history and mating systems shape TE diversity. However, the exact way in which they contribute to TE genome distribution in Drosophila remains obscure, particularly because of the lack of comparative studies on parallel populations submitted to similar environment and/or population conditions. In particular, colonising populations subjected to genetic drift processes due to drastic decreases in population size (i.e. bottlenecks), or confronted by new ecological conditions, could constitute an excellent model for carrying out these studies and evaluate the effect of demography versus selection.

Drosophila buzzatii, a cactophilic species that originated in the Chaco region of NW Argentina, colonised the Old World and Australia 300 and 70 years ago, respectively. Whereas the former colonisation was probably associated with South American colonial trade and is not well documented, highly documented information is available for the Australian colonisation. The introduction of D. buzzatii into Australia is associated with the program of biological control of the prickly pear (Opuntia sp.) infestation that devastated Australian agricultural crops in the 1920s [8]. D. buzzatii was most probably introduced into Australia inadvertently via rotten Opuntia cladodes containing larvae of Cactoblastis cactorum, a natural moth parasite of Opuntia, largely being transported in shipments from Argentina, that was used for Opuntia pest control. Cactoblastis and the accompanying insects spread rapidly to all Opuntiainfested fields $[8,9]$. After the plague was controlled, Opuntia diminished drastically in population size, remaining established in scattered habitat patches [10]. Our previous studies concerning Old World D. buzzatii colonisation demonstrated a bimodal pattern of distribution of the Osvaldo retrotransposon consisting of high and low insertion frequency sites. However, it is unknown whether this distribution pattern, valid for the Mediterranean colonisation that occurred three centuries ago, is valid for other colonisation processes. The aim of the present work is to study the genome distribution of two retrotransposons, Osvaldo [11] and Isis [12], in some of these colonising Australian populations of D. buzzatii. Subsequently, these results will be compared to those of previous studies concerning the retrotransposon Osvaldo in colonising populations of the Iberian Peninsula and in the original Argentina populations in order to evaluate how far TE dynamics depend on population parameters inherent in colonisation processes. Our results show that Australian populations have a mixture of high and low insertion frequency sites for both TEs and the drift effect associated to colonisation is probably the main factor responsible for the TEs distribution frequency. High insertion frequency sites would represent insertions prior to the colonisation. Sites of low frequency would correspond to new insertions occurred after the colonisation.

\section{Results}

Genomic distribution of Osvaldo and Isis retrotransposons The distribution of Osvaldo and Isis was analysed in the whole genome of two D. buzzatii Australian natural populations. The shape of the chromosomal distribution of these TEs is depicted in Figure 1; Osvaldo and Isis distributions are represented on chromomes 2 and 3, respectively, as an example. Both TE distributions are characterised by two kinds of sites: sites with low and sites with high $(>10 \%)$ insertion frequencies. This pattern was not observed for Osvaldo on other chromosomes, where the frequency of the highest occupied sites never reached 10\% (data not shown). In the case of Isis, the same pattern is observed on chromosomes 2 and 5 , with occupancy frequencies up to $30 \%$ in some sites. Osvaldo has three (2B2a, 2D5a and $2 \mathrm{G} 2 \mathrm{f})$ and Isis five (2B2a, 2E5a, 3B5f, 3E5b, 5G1a) high occupancy sites in the whole genome. The occupancy (number of times that a site is present in the population) profiles of the two elements are similar in the two populations studied, but Isis has a slightly higher occupancy frequency than Osvaldo (Table 1). Table 2 summarizes the means and variances of copy number per haploid genome for the two retrotransposons. The mean copy number is low for both elements in the two populations but higher for Isis than for Osvaldo. Overall mean copy number comparisons between chromosomes demonstrates that whereas the $\mathrm{X}$ chromosome presents the lowest mean copy number for both elements, chromosomes 2 and 3 harbor the highest mean numbers of Osvaldo and Isis copies, respectively. Deviations from Poisson distribution were tested using chi-square goodness of fit, pooling adjacent classes with low expected copy numbers.

In order to test whether the TE insertions are distributed in a repulsive (rarely inserted in nearby regions) or a contagious (frequently inserted in nearby regions) way in each chromosome separately, we computed the linkage disequilibrium for each pair of sites detected by analysing each possible $2 \times 2$ contingency table [13]. The observed distribution of disequilibria was compared with the expected distribution (assuming no linkage disequilibrium) using Fisher's hypergeometric formula [14]. A battery of correlation tests was performed for each transposable element and chromosomal arm, and the values were pooled at intervals of 0.1 . Figure 2 depicts the two tests that 


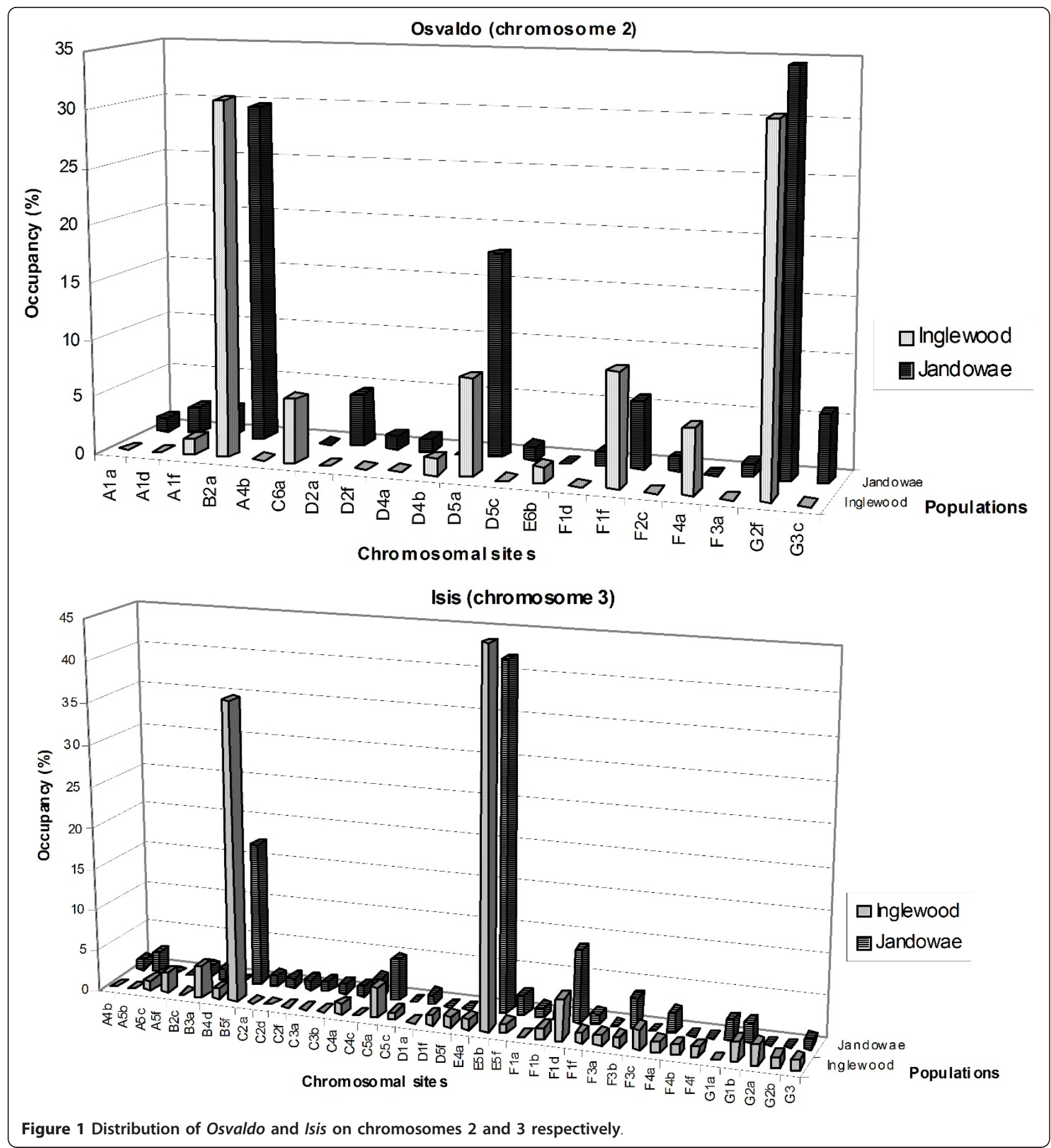

produced significant values and represents observed and expected correlation distributions of Osvaldo and Isis on chromosome 3 of the Jandowae population. Tests were significant for the two elements owing to an overabundance of classes with positive correlation coefficients. In the case of Isis, an excess of the class -0.19;-0.10 is observed, but the test is within the limits of significance $(\mathrm{P}=0.04)$
Comparative study of Osvaldo and Isis copy numbers on different chromosomes

The factors controlling TEs in natural populations and the magnitude of their impact on host genomes are topics of much discussion. The genomic distribution of TEs has been interpreted as the result of negative selection against their deleterious effects [3]. Negative selection could act either against deleterious effects of 
Table 1 Occupancy profiles of euchromatic sites in Australian colonising populations

\begin{tabular}{ccccccccccccccccccccccc}
\hline \multicolumn{1}{c}{ TE } & Populations & \multicolumn{11}{c}{ Occupancy profiles } \\
\hline \multirow{3}{*}{ Osvaldo } & & 1 & 2 & 3 & 4 & 5 & 6 & 7 & 8 & 10 & 14 & 15 & 22 & 23 & 25 & 28 & 29 & 34 & 36 \\
& IN & 37 & 5 & 1 & 3 & 0 & 1 & 1 & 0 & 0 & 0 & 0 & 1 & 1 & 0 & 0 & 0 & 0 & 0 \\
& JA & 33 & 10 & 2 & 1 & 3 & 1 & 0 & 0 & 0 & 0 & 1 & 0 & 0 & 1 & 0 & 1 & 0 & 0 \\
\hline \multirow{2}{*}{ Isis } & IN & 47 & 12 & 1 & 4 & 3 & 3 & 1 & 2 & 2 & 1 & 0 & 0 & 0 & 0 & 1 & 0 & 1 & 0 \\
& JA & 47 & 14 & 7 & 2 & 0 & 1 & 2 & 0 & 0 & 0 & 1 & 0 & 0 & 1 & 0 & 1 & 0 & 1 \\
\hline
\end{tabular}

Population origin: Inglewood (IN) and Jandowae (JA).

Occupancy profiles: number of times that each site is occupied in a population. For example, in the Inglewood population there are 37 sites occupied once, five sites twice, etc.

insertions and/or of chromosomal rearrangements resulting from ectopic crossing over between TE copies [2]. If selection plays a preponderant role in TE distribution, fewer insertions should be present on the X chromosome than in autosomes because of the deleterious effects on the $\mathrm{X}$ chromosome in hemizygous males [3]. In order to investigate whether this negative selection affected the Osvaldo and Isis distribution in Australian populations, their copy numbers were compared between chromosome $\mathrm{X}$ and autosomes. Observed and expected proportions were compared using a $\mathrm{G}$ test [13] among autosomes $(\mathrm{Gc})$, between $\mathrm{X}$ and autosomes $(\mathrm{Gb})$ and among all chromosomes (Ga). It was assumed that the expected number of copies was proportional to the relative amount of DNA in each chromosome. The number of bands per chromosome in the cytological map of D. buzzatii [15] was considered directly proportional to the amount of DNA, as per previous studies

Table 2 Test of the Poisson distribution of Osvaldo and Isis per chromosome and haploid genome

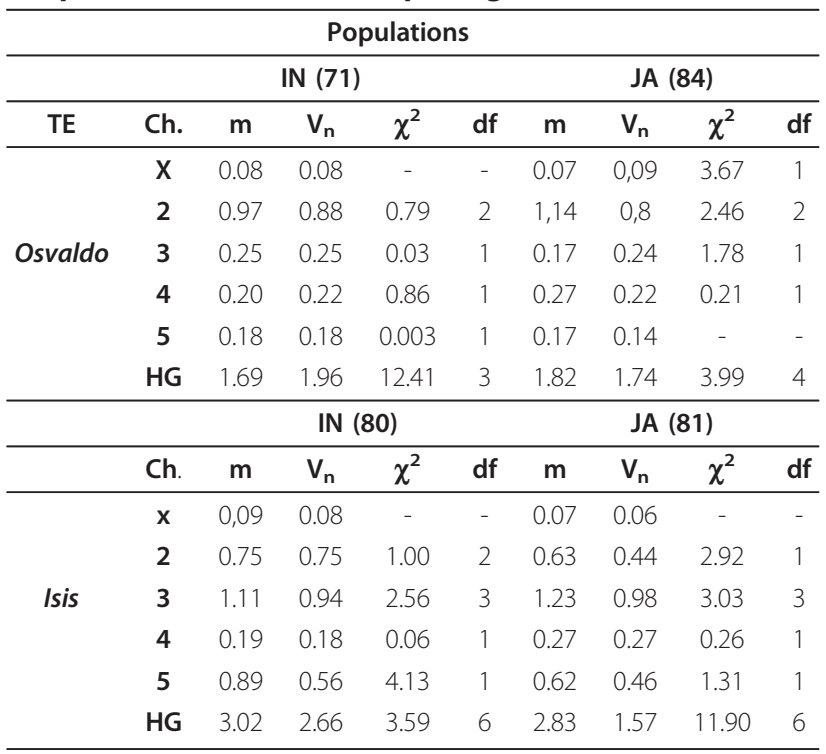

See Table 1 for population identification. TEs: transposable elements; $\mathrm{Ch}$ : chromosome; HG: haploid genome; $m$ : mean copy number; $V_{n}$ : variance of copy number; df: degrees of freedom; Bonferroni's correction was applied to the data. ${ }^{*} P<0.05 ;{ }^{*} P<0.01$. The numbers of individuals analyzed are indicated in parenthesis.
$[16,17]$. To estimate the expected TE proportions, the proportion of bands of each chromosome was multiplied by the total TE copy number detected in a population. The results of the G test, depicted in Table 3, demonstrate that differences in Osvaldo between chromosome $\mathrm{X}$ and autosomes are significant when sites of high insertion frequency are considered but not significant when these sites are omitted from the analysis. However, Isis appears to be more strongly controlled by natural selection than Osvaldo because the results of comparisons between chromosome $\mathrm{X}$ and autosomes were significant regardless of whether high insertion sites were considered. This element is characterised by lower and higher copy numbers on chromosomes $\mathrm{X}$ and

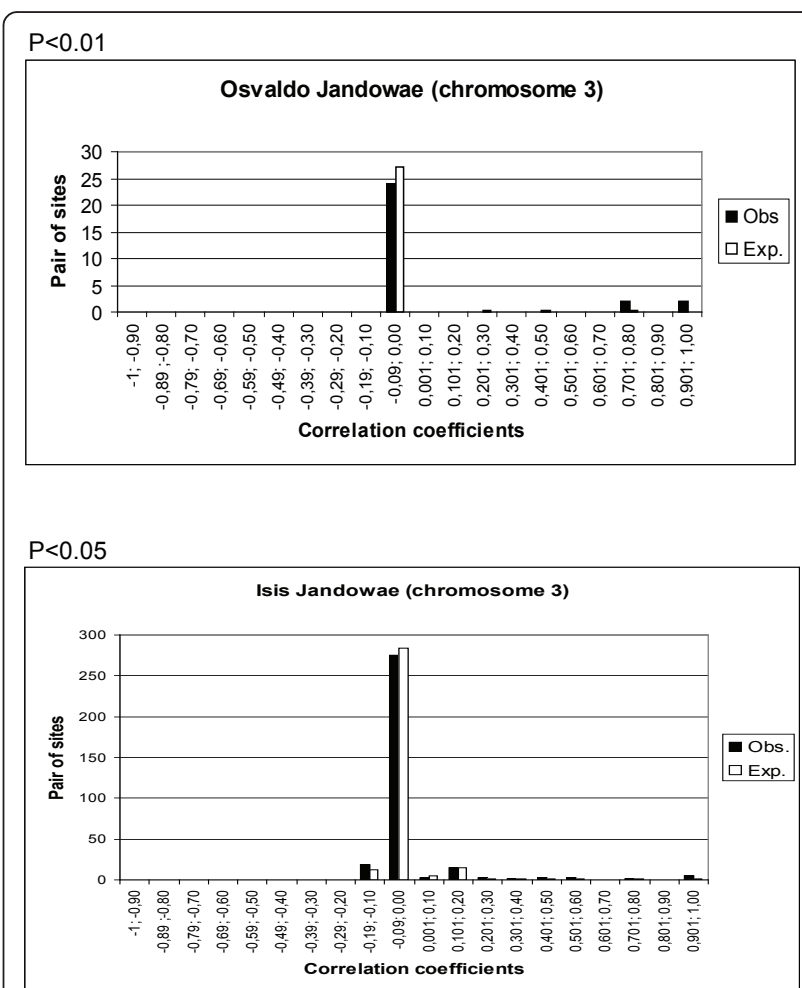

Figure 2 Observed and expected frequency distributions of correlation coefficients between all pairs of sites of chromosome 3 in Jandowae population. A) Osvaldo; B) Isis 
Table 3 Comparisons of the proportion of Osvaldo and Isis among chromosomes

\begin{tabular}{|c|c|c|c|c|c|}
\hline \multirow[b]{2}{*}{ Chromosomes } & \multirow[b]{2}{*}{ Exp. Prop. } & \multicolumn{2}{|c|}{ Osvaldo } & \multicolumn{2}{|c|}{ Isis } \\
\hline & & IN & JA & IN & JA \\
\hline$x$ & 0.156 & 0.05 & 0.04 & 0.03 & 0.02 \\
\hline 2 & 0.253 & 0.58 & 0.63 & 0.25 & 0.22 \\
\hline 3 & 0.207 & 0.15 & 0.09 & 0.37 & 0.44 \\
\hline 4 & 0.186 & 0.12 & 0.15 & 0.06 & 0.10 \\
\hline \multirow[t]{2}{*}{5} & 0.197 & 0.11 & 0.09 & 0,29 & 0.22 \\
\hline & Df & & & & \\
\hline \multirow[t]{2}{*}{$\mathrm{Ga}$} & 4 & $59.44^{* *}$ & $130.70^{* *}$ & $99.87^{* *}$ & $96.09^{* *}$ \\
\hline & & (5.36) & $(10.59)$ & $(30.49 * *)$ & $\left(32.13^{* *}\right)$ \\
\hline \multirow[t]{2}{*}{ Gb } & 1 & $13.32^{* *}$ & $21.53^{* *}$ & $42.32^{* *}$ & $42.39 * *$ \\
\hline & & $(3.88)$ & $(5.54)$ & $\left(16.49^{* *}\right)$ & $\left(18.46^{* *}\right)$ \\
\hline \multirow[t]{2}{*}{ Gc } & 3 & $46.12^{* *}$ & $82.16^{* *}$ & $57.55^{* *}$ & $53.70^{* *}$ \\
\hline & & $(1.47)$ & $(5.05)$ & $(14.00)$ & $\left(13.67^{*}\right)$ \\
\hline \multicolumn{6}{|l|}{$\mathrm{Ga}$} \\
\hline Total & 8 & \multicolumn{2}{|c|}{$163.13^{* *}(15.95)$} & \multicolumn{2}{|c|}{$195.97^{* *}\left(62.62^{* *}\right)$} \\
\hline Pooled & 4 & \multicolumn{2}{|c|}{$159.94^{* *}\left(13.53^{* *}\right)$} & \multicolumn{2}{|c|}{$189.88^{* *}\left(54.35^{* *}\right)$} \\
\hline $\mathrm{H}$ & 4 & \multicolumn{2}{|c|}{$3.19(2.42)$} & \multicolumn{2}{|c|}{$6.08(8.27)$} \\
\hline \multicolumn{6}{|l|}{$\mathrm{Gb}$} \\
\hline Total & 2 & \multicolumn{2}{|c|}{$34.85^{* *}\left(9.42^{*}\right)$} & \multicolumn{2}{|c|}{$84.71^{* *}\left(34.95^{* *}\right)$} \\
\hline Pooled & 1 & \multicolumn{2}{|c|}{$34.67^{* *}\left(9.37^{* *}\right)$} & \multicolumn{2}{|c|}{$84.64^{* *}\left(34.89^{* *}\right)$} \\
\hline $\mathrm{H}$ & 1 & \multicolumn{2}{|c|}{$0.18(0.009)$} & \multicolumn{2}{|c|}{$0.08(0.06)$} \\
\hline \multicolumn{6}{|l|}{ Gc } \\
\hline Total & 6 & \multicolumn{2}{|c|}{$128.28^{* *}(6.53)$} & \multicolumn{2}{|c|}{$111.25^{* *}\left(27.67^{* *}\right)$} \\
\hline Pooled & 3 & \multicolumn{2}{|c|}{$125.28^{* *}(4.17)$} & \multicolumn{2}{|c|}{$105.25^{* *}\left(19.46^{* *}\right)$} \\
\hline $\mathrm{H}$ & 3 & \multicolumn{2}{|c|}{$3.0(2.36)$} & \multicolumn{2}{|c|}{$6.00(8.21)$} \\
\hline
\end{tabular}

Exp. Prop.: Expected TE proportions; Ga: Comparison of the proportion of TEs among chromosomes; Gb: Comparison of the proportion of TEs between chromosome $\mathrm{X}$ and autosomes; Gc: Comparison of the proportion of TEs among autosomes; $\mathrm{H}$ : Heterogeneity; Df: degrees of freedom;*P $<0.05 ;{ }^{* * P}<$ 0.01; Bonferroni's correction was applied. Values of the test excluding high insertion frequency sites are indicated in parenthesis.

3 , respectively, than would be expected under a random genome distribution. A heterogeneity test [13] was carried out to determine whether the differences always occur in the same direction and the results demonstrate that the two populations generally do not differ for Isis and Osvaldo copy numbers over the chromosomes. Comparisons among autosomes, when the two populations are pooled and sites of high insertion frequency are eliminated, gave significant results for the Isis element but not for Osvaldo.

\section{Inversion polymorphism in Australian populations of D. buzzatii}

The original South American populations of D. buzzatii present inversion polymorphisms on chromosomes 2 and 4 , with eight $\left(2 \mathrm{st}, 2 \mathrm{j}, 2 \mathrm{jz}^{3}, 2 \mathrm{jq}^{7}, 2 \mathrm{y}^{3}, 2 \mathrm{c}^{9}, 2 \mathrm{r}^{9}, 2 \mathrm{js} \mathrm{s}^{9}\right)$ and two (4s and $4 \mathrm{st}$ ) chromosome arrangements, respectively [18]. As a result of the colonising processes in Australia and the Old World, rare arrangements were lost and others have changed their frequency. The two Australian populations considered in this study present two arrangements in chromosome 2: st and j, which are also the most frequent in the original and the Old World populations. The frequencies of arrangements st and $j$ are 0.40 and 0.60 in Inglewood and 0.36 and 0.64 in Jandowae, respectively. In contrast to the Old World colonisation, arrangements $2 \mathrm{jq}^{7}$ and $4 \mathrm{~s}$ have never been detected in Australia [19]. Interestingly, the 2jz3 arrangement, absent in our samples, was identified in other studies at a very low frequency [20].

Recombination has been considered the main factor responsible for the distribution of TEs on chromosomes $[21,22]$. According to the model proposed by Langley et al. [23], TEs can induce chromosomal rearrangements by ectopic crossing-over between homologous elements located in different chromosomal sites. If the model predictions are true, we will find TE accumulation in regions of low recombination such as inside inversions and adjacent to inversion break-points. In order to determine whether the arrangements are associated with high insertion sites for Osvaldo and Isis, we computed the productmoment correlation coefficients (r). The results depicted in table 4 demonstrate a significant negative correlation between several highly occupied sites of Osvaldo (2D5a and 2G2f) and the $j$ arrangement in Jandowae. Interestingly, in the Inglewood population, correlation coefficients for Isis and Osvaldo have the same sign (except for the site 2G2f of Osvaldo) for both elements, but neither is significant. Moreover, of the sites that have a significant correlation, only one is located inside the $\mathrm{j}$ inversion (2D5a); the other is located in its break point (2E5a).

\section{Comparisons of TE mean copy numbers between} Australian and Old World colonisations of $D$. buzzatii The Old World colonisation by D. buzzatii has been studied intensively in our laboratory with the Osvaldo TE $[16,17]$. We combined these data with those from

Table 4 Correlation coefficients between J chromosomal arrangement and high insertion frequency (HF) sites

\begin{tabular}{cccc}
\hline & & \multicolumn{2}{c}{ Populations } \\
\hline & Arrangement & IN & JA \\
\hline HF sites of Osvaldo & & Corr. & Corr. \\
2B2a & $J$ & 0.02 & 0.12 \\
$2 \mathrm{D} 5 \mathrm{a}$ & $\mathrm{J}$ & -0.15 & $-0.30^{* *}$ \\
$2 \mathrm{G} 2 \mathrm{f}$ & $\mathrm{J}$ & 0.08 & $-0.29^{* *}$ \\
HF sites of Isis & & & \\
$2 \mathrm{2} 2 \mathrm{a}$ & $\mathrm{J}$ & -0.04 & $-0,21$ \\
$2 \mathrm{E} 5 \mathrm{a}$ & $\mathrm{J}$ & 0,09 & 0.02 \\
& & & \\
\hline
\end{tabular}

Corr: correlation coefficient. Only sites having a frequency $\geq 0.10$ were considered. 
the Australia colonisation to compare mean Osvaldo copy numbers between both colonising populations and the original ones. Table 5 summarizes the mean Osvaldo copy numbers in the natural populations from Argentina and the Iberian Peninsula studied previously, and those from Australia. We observed that the original Argentinean populations have the lowest mean copy number of Osvaldo, with a slight difference between the two sets of populations analysed in two previous works $[16,17]$. Colonising populations from the Iberian Peninsula possessed the highest mean copy number. A Kruskal and Wallis non-parametric test of analysis of variance was performed to investigate whether the mean copy number of Osvaldo differs among populations from a given area and between populations from different areas (Figure 3A). While no differences in copy number were observed among Australian populations $(\mathrm{H}=0.53, \mathrm{P}=0.46)$ or among Iberian Peninsula populations $(\mathrm{H}=5.45, \mathrm{P}=0.36)$, there were significant differences among populations from Argentina $\left(H=16.17^{*}\right.$, $\mathrm{P}=0.02)$. When the three population groups were compared, the differences were significant $\left(\mathrm{H}=219.84^{* *}\right.$, $\mathrm{P}=0.00$ ) and the Australian populations had mean copy number values intermediate between the Argentinean and Old World populations. The same analysis was carried out excluding positions of high insertion frequency in the colonising populations (see Figure 3B); the significance disappeared in all cases except when Argentinean and Old World populations were compared $(\mathrm{H}=7.27 * \%, \mathrm{P}=0.007)$. We conclude that the high mean copy number in colonising Australian populations can only be explained by the presence of some sites with high insertion frequency. Colonising populations could have suffered a genomic redistribution of the Osvaldo retrotransposon, probably due to the founder effect. As a consequence, certain copies arriving with the founders have a high occupancy in the current populations. These results agree with the hypothesis that the mean copy number of Osvaldo increases during the colonisation process. However, in the case of Old World colonising populations, differences still persist after elimination of high insertion frequency sites. The most likely explanation for this is the large mean copy number heterogeneity among Argentinean populations, because when the two populations with the lowest copy numbers are eliminated from the comparison, the differences become insignificant. In the case of Isis, a recently characterised element, no information is available from previous population studies, but the mean copy number of laboratory lines from Spanish populations is approximately three copies per genome [12], a value similar to that found in Australian populations.

The sites with the highest insertion frequency of Osvaldo in different colonising populations from the Iberian Peninsula and Australia are presented in Table 6. There was a general tendency towards lower numbers of highly occupied Osvaldo sites in Australia than in the Iberian Peninsula.

\section{Discussion}

TE copy numbers and distribution: Australia vs. Old World colonisations

Comparing this study with previous works that analysed original (from Argentina) and colonising (from the Iberian Peninsula and Australia) populations, some differences are apparent. While the original populations always demonstrate low occupancy per chromosomal site, the colonising populations (Australian and Spanish) have some sites with high occupancy. When colonising populations are compared, Old World populations have more highly occupied Osvaldo sites (eight) and a higher occupancy rate of some sites (up to 63\% occupancy) than the Australian populations (three sites with less than 33\% occupancy). The mean copy number is lower in Australian than in Old World populations, placing Australia in an intermediate position between Argentina and the Old World. Data from Isis are limited to the Australian populations, but interestingly, the mean copy number is similar in Australian and Old World laboratory stocks.

The overall picture that emerges from these data suggests a global increase in the mean copy number per

Table 5 Mean copy number of Osvaldo retrotransposon in different natural $D$. buzzatii populations: original and colonising

\begin{tabular}{|c|c|c|c|c|c|}
\hline \multirow{3}{*}{$\begin{array}{l}\text { ELEMENT } \\
\text { Osvaldo }\end{array}$} & & \multicolumn{4}{|c|}{ POPULATIONS } \\
\hline & \multicolumn{2}{|c|}{ Original } & \multicolumn{3}{|c|}{ Colonising } \\
\hline & $\begin{array}{c}\text { Argentina } a_{a} \\
(6)\end{array}$ & $\begin{array}{l}\text { Argentina } a_{b} \\
(2)\end{array}$ & I. Peninsula ${ }_{a}$ & $\begin{array}{l}\text { I. Peninsula } \\
\text { (1) }\end{array}$ & $\begin{array}{c}\text { Australia } \\
\text { (2) }\end{array}$ \\
\hline Mean & 1.22 & 0.85 & 2.85 & 2.99 & 1.75 \\
\hline
\end{tabular}

The numbers of populations analyzed are in parenthesis; subscripts represent a detailed explanation about populations and bibliographic references; Argentinean populations (Amaicha, Arroyo Escobar, Catamarca, Cébila, Palo Labrado and Vipos) and Iberian populations (Carboneras, Sanlúcar, La línea, Mazarrón and Albufeira) as described in Labrador et al. 1998; : Argentinean populations of Berna and Puerto Tirol and Iberian population of Carboneras, as described in García Guerreiro and Fontdevila; : Australian populations (Inglewood and Jandowae) presented in this work. 


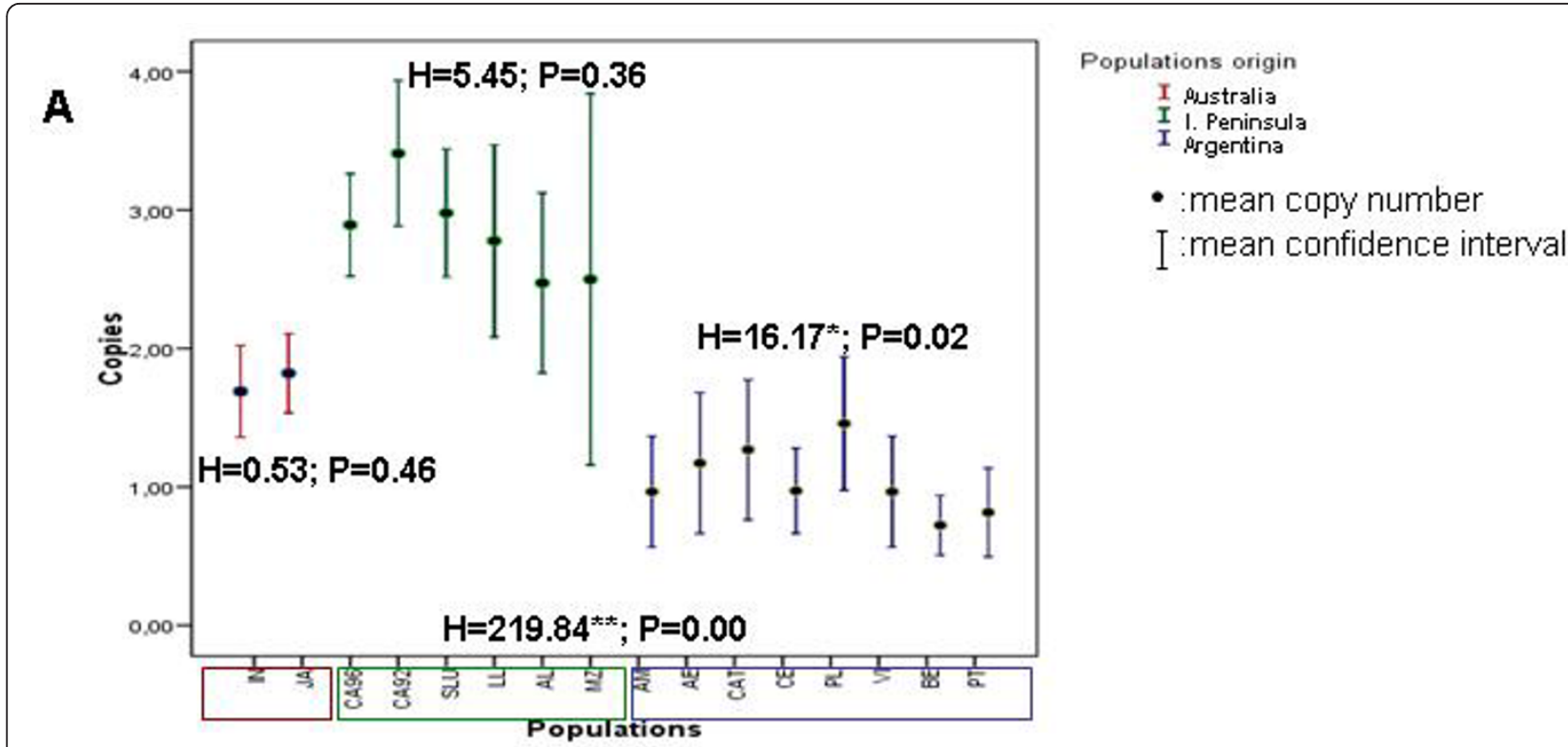

$\mathrm{H}=28.82 ; \mathrm{P}=0.02$

B

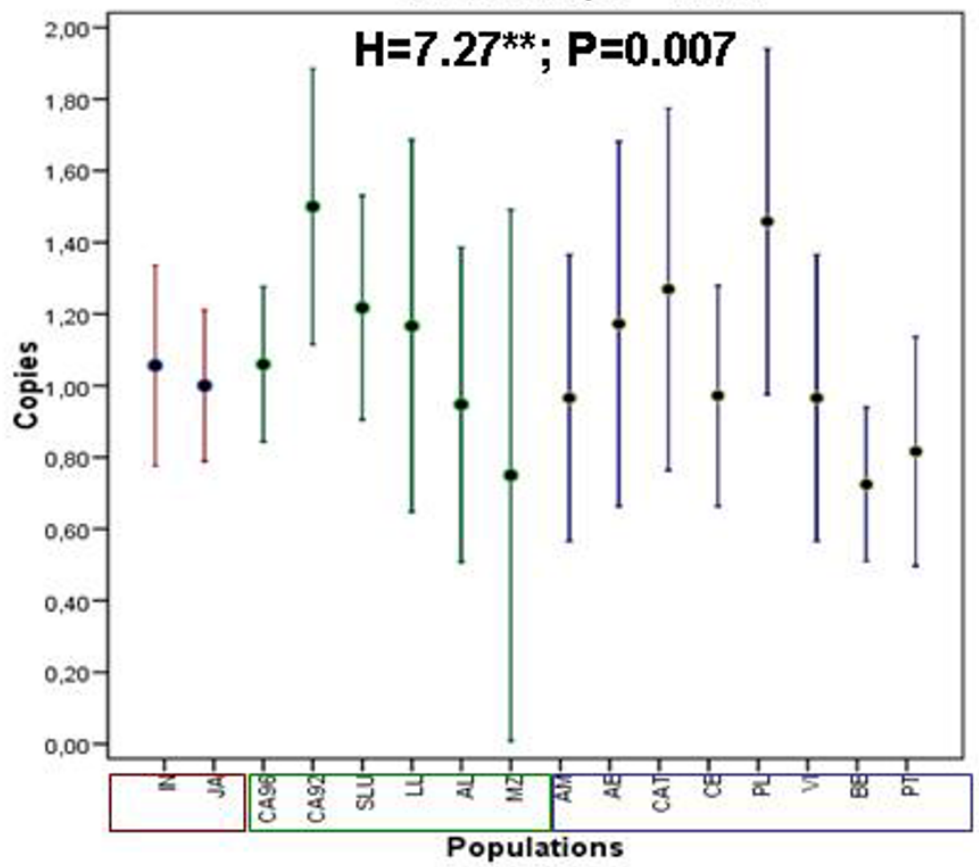

Figure 3 Kruskal-Wallis non parametric test of analysis of variance for the mean copy number of Osvaldo. A) All sites included, B) Excluding high insertion frequency sites. Populations identification: JA (Jandowae), IN(Inglewood), Argentinean and Spanish populations are explained in references $[16,17]$.

site of Osvaldo during the colonisation process. This may be explained either by the drift effect following the founder event acting on many low occupied sites (turning them into high occupied sites) or by increases of site-directed transposition rates in colonisation, or both. According to previous work $[16,17,24,25]$, sites demonstrating high insertion frequencies are most likely due to a founder effect that occurred during the colonisation process, and low insertion sites are probably the result of new transposition events.

The Australian and Spanish populations have similar insertion-site distribution patterns but some of the 
Table 6 The highest frequencies of Osvaldo insertion sites in two groups of colonising populations

\begin{tabular}{ccc}
\hline High frequency sites & I. Península $\left.\mathrm{a}_{\mathbf{a}} \mathbf{6}\right)$ & Australia $_{\mathbf{b}}$ (2) \\
\hline 2B2a & 0.48 & 0.31 \\
2D5a & & 0.18 \\
2D1h & 0.22 & \\
2F4a & 0.42 & \\
2F1fg & 0.18 & 0.32 \\
2G2f & & \\
2G2h & 0.26 & \\
3F2b & 0.16 & \\
4C2g & 0.10 & \\
5A4b & 0.63 & \\
\hline
\end{tabular}

The numbers of populations considered are in parenthesis; subscripts

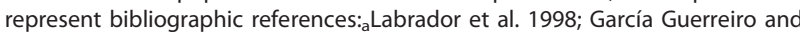
Fontdevila, this work. See Table 5 for population identification. Only sites having a frequency $\geq 0.10$ are presented.

aforementioned differences deserve more detailed analysis. First, high insertion frequency sites are generally located in different chromosomal positions in the two colonisations, indicating two independent colonisation events from Argentina. Second, the number of highly occupied sites is greater in the Spanish than in the Australian colonising populations. These differences could be related to specific differences in the two colonising processes. While Old World colonisation appears to be caused by a low number of founders [17], the number of $D$. buzzatii individuals introduced during the Australian colonisation was certainly large initially $[8,9]$ but fell dramatically following the Opuntia biological control program. Therefore, we can imagine a scenario where D. buzzatii spread rapidly through the Opuntia area until 200,000 hectares of prickley-pear had been largely destroyed by biological control [26]. Since D. buzzatii is restricted to the Opuntia distribution area [27], a parallel drastic decrease of the D. buzzatii population size should have occurred. This rapid reduction in the number of coloniser survivors could explain, at least in part, why the number of highly occupied sites in Osvaldo is lower in the Australian colonisation than in the Old World one.

The results showed here are in accordance with previous comparisons using allozyme variability $[19,28]$, microsatellites [10,29] and mitochondrial DNA [30], where a decrease in variation was observed in Australian populations, probably due to a bottleneck followed by a population expansion after the initial founder event. Though the founder effect hypothesis is widely accepted by all authors working on Australian D. buzzatii populations, there are discrepancies in relation to the bottleneck magnitude depending on the markers used. Whereas nuclear markers such as microsatellites [10] and allozymes [31] suggest a moderate bottleneck, mitochondrial
DNA [30] data point to a very low number of founders. The mtDNA was considered a particularly valuable marker for studies of recent population history owing to its maternal inheritance, lack of recombination and rapid evolution (relative to nuclear genes) $[32,33]$. The fact that data concerning TE distribution tend to be more in agreement with mitochondrial data, and that records of the cactus control program implied a strict quarantine of associated insects, suggest that the most likely explanation is that only a few individuals founded the Australian populations. Therefore, it is expected that the magnitude of the founder effect was stronger in Australian populations than in Old World populations

On the other hand, during colonisation selection could also play un important role for the elimination of deleterious TE insertions. For example, homozygosity can increase during the colonisation process, if a few colonisers are involved in the process, increasing the strength of selection against deleterious insertions as observed in selfing plant species [34,35] Additionally, selection could contribute to the adaptation to new environments as seen during the expansion of $D$. melanogaster from Africa [36,37]. Recent results demonstrate a role for some TEs in the adaptation of D. melanogaster colonising populations to the out of Africa temperate climate [38]. It is not impossible that some insertions are maintained because of their positive effects in terms of adaptation to the new environments encountered by colonisers. The bottleneck suffered by Australian populations could have produced gene frequency shifts that have been proposed as responsible for driving populations to new adaptive peaks by selection. But, in the present study, the presence of high insertion sites is more likely to be due to the drift effect associated to the colonisation process because the Australian D. buzzatii colonisation time of approximately 840 generations (70 years multiplied by 12 generations per year) is probably insufficient to detect selection effects unequivocally. This hypothesis is supported by results in previous studies where the Osvaldo DNA altered sequence and its flanking genomic sequences in high frequency sites were identical in all Old World colonising populations [24], a result highly improbable under the selective hypothesis Thus, when considering all results together we can conclude that selection does not seem to play a major role, compared to demography, in the distribution of transposable elements in the Australian colonising populations.

\section{TE dynamics in the colonisation processes}

Colonisation has been suggested as one of the processes responsible for awakening transposable elements in D. simulans [39]. During colonisation, individuals are exposed to stress attributable to new environmental conditions and population regimes. Environmental stresses can 
induce changes in chromatin structure [1] and promote TE mobilisation as observed in plants [40] and other organisms [41]. D. buzzatii, a cactophilic species, has specific resource requirements that imply intense natural selection for survival in extreme environments. Therefore, these species are less able to avoid environmental stress by habitat selection [42].

Considering a very simple model of colonisation, transposition rates in colonisers can be estimated. We assume that sites originating from Argentina during colonisation are found at high frequency in colonising populations and that new transpositions correspond to low frequency sites. The estimations begin with the historical data, which suggest that Australian colonisation of D. buzzatii occurred approximately 70 years ago $[31,43]$ and assume a mean of 12 generations per year. Taking the pooled data from the two Australian populations (155 genomes), we estimate 840 generations of colonisation (70 years multiplied by 12 generations), 93 new sites and a mean copy number for Osvaldo insertions of 1.75 . We estimate a minimum value of transposition rate per site per generation in colonisers, for Osvaldo, of $4.08 \times 10^{-4}(93 / 155 \times 1 / 840 \times 1.75)$ and for Isis of $3.62 \times 10^{-4}$. These values have the same order of magnitude as those calculated for Osvaldo $\left(1.28 \times 10^{-4}\right)$ in previous studies with natural populations from the Old World [17]. No differences in transposition rates were observed between the two colonisations, or between the two transposable elements considered in this study.

We cannot completely disregard local transposition events in some coloniser populations. It is well known that transpositional bursts have occasionally been found in Drosophila melanogaster laboratory stocks [44], other Drosophila species [45] and in plants subjected to different environmental stresses [46]. However, transposition bursts are difficult to see in nature in real time because TE copies involved in these events are later silenced owing to their deleterious effect to the host. The majority of these mechanisms include host factors as methylation [47], deletions [48], silencing by RNA interference [49] or random mutations, excisions and purifying selection [50].

In the case of $D$. buzzatii colonisation processes, we can accept that the populations analysed have not suffered large transposition rate changes. Another possibility is to imagine that even if colonisation induced local transposition it could be unnoticed owing to the time elapsed, especially if the TE eliminations are quick, as observed in the Helena element in genomes of D. melanogaster and D. simulans [48]. Old World colonising populations of D. buzzatii contain TEs where active and inactive copies coexist [24] and the low occupancy sites observed could be the result of a unique transposition or very few master copies present in the genome. However, the possibility that some low occupied sites harbored copies that have diminished their frequency in populations because of drift or selection effects against insertions with a deleterious effect cannot be disregarded. A colonising population from the Old World (Carboneras) demonstrated a decrease in the insertion frequency of many highly occupied sites (2B2a, 2F4a). In other studies concerning D. subobscura colonising species, the existence of common low frequency sites of bilbo and gypsy elements was attributed to a decrease in insertion frequency in some sites in coloniser populations [25]. However, we hypothesize that high occupancy sites correspond to sites that have increased their frequency by drift associated to the colonisation process. Arguments in favor of this hypothesis are that sites of both elements, found at a low frequency in the original populations, are now at a high frequency in populations resulting from the two colonisations. However, a common highly occupied site (2B2a) of Osvaldo and Isis elements was observed in the Australian populations. This site could be a transposition hot spot induced by the colonisation process as it is present at a very low frequency in some original populations. Moreover, the two elements are inserted in the same chromosomal band, confirming previous results where Isis retrotransposon appeared to have preferential insertions inside Osvaldo sequences [12].

Comparisons of TE copy numbers between chromosome $\mathrm{X}$ and autosomes, after elimination of high insertion sites, demonstrated that Isis appears to be controlled by purifying selection in Australian populations, whereas the selection effect is not so evident for Osvaldo. However, the comparison is highly significant when the two Australian populations are pooled, which may suggest a stronger selection intensity against Osvaldo insertions in these populations. These differences may be due to a stronger selection effect in the Australian colonisation than the Old World one. Alternatively, the differences observed between the two elements could be due to the existence of differential transposition and regulation mechanisms. Another explanation may be that selection effects on the Osvaldo element could go unnoticed if transposition events occurred only a few generations ago. A third possibility is that selection acts more strongly on the Isis element (for example, if the element is inserted inside genes or regulatory regions) than on Osvaldo and, provided the Australian colonisation is recent, its effects are only detected in the element under the most selection pressure.

Comparisons of the proportion of elements between autosomes demonstrated large differences due to an overrepresentation of Osvaldo on chromosome 2 (58-63\% from the total) and of Isis on chromosome 3 (37-44\% from the total). D. buzzatii Australian populations have one inversion on chromosome 2. Inversions [23,51], inversion break-points and nearby regions [52-54] are considered by some authors as sites of accumulation of TEs. These regions are characterised by a reduction in recombination 
rates that diminishes the probability of deleterious ectopic exchanges. Correlations between highly occupied sites of Osvaldo and the J arrangement produce negative values; only two sites were significant: one located outside and another inside the J inversion. The most likely explanation is that most correlations observed are a consequence of the founder effect. This would explain why significant negative correlations are observed even in sites located inside the inversion. A similar interpretation was evident in a study concerning colonising $D$. subobscura populations, where the founder hypothesis was favored by the fact that all correlations between sites and arrangements were significant only in the colonising populations [25]. Moreover, there were positive associations between chromosomal arrangements and highly occupied sites located outside of inversions. This result is not unexpected if we take into account that the effective population size is reduced during the first stages of colonisation. If the TE abundance is regulated by the effect of ectopic recombination between elements [2], the existence of insertions in a homozygous state could reduce ectopic exchange events $[35,55]$ outside inversions, leading to a relaxation of selection pressure.

\section{Conclusions}

Knowledge concerning TE distribution in natural populations, subjected to different environmental and demographic regimes, is an important step towards understanding their role in species evolution. Here we demonstrate that the two retrotransposons studied in Australian populations presented bimodal distribution with high and low insertion frequency sites. These results indicate that Australian D. buzzatii populations were subjected to a founder effect and a strong bottleneck during the colonisation process. This work emphasizes the importance of population factors in remodelling the distribution of TEs in the genomes of natural populations.

\section{Methods}

\section{Drosophila strains and mating system}

Two natural populations from Southeast Australia: Inglewood $\left(31.55^{\circ} \mathrm{S} / 151.52^{\circ} \mathrm{E}\right)$ and Jandowae $\left(26.46^{\circ} \mathrm{S} / 151.50^{\circ}\right.$ E), were caught in March 2006. Of the individuals collected, approximately one hundred males and 15 females of $D$. buzzatii were checked. Immediately after their arrival at the laboratory each wild male was crossed with three virgin females from the control line 63 42/7 F81 [24]. Isofemale lines were established from each wild female and subsequently one F1 male crossed in the same way as described for wild males. The control line was checked by in situ hybridisation before the experiment and was characterised as devoid of euchromatic copies of Osvaldo bearing two copies of Isis element (2F1d and 4F2b), with constant insertion profile over different generations.

Only female larvae were analysed in crosses with the control line in order to have the whole wild haploid genome, including X chromosome. The Isis insertion profile of each individual was obtained by subtracting the insertion profile of the control line from that of the F1 larvae. The minor disadvantage of using this method is that, in the case of Isis, the putative common sites between wild individuals and the control line were omitted from our studies. However, this is a powerful and reliable method used in previous studies concerning transposable element distribution in D. buzzatii and D. subobscura $[16,17,25]$

\section{DNA probes}

A $6.4 \mathrm{~kb}$ fragment of the Osvaldo retrotransposon, including the main part of the complete element except the two LTRs, was used and cloned in the pBSK vector. The Isis probe consisted of a $3.4 \mathrm{~kb}$ PCR fragment including pol-env [12] inserted in the pGEM-T vector (Promega). Both probes were labeled with digoxigenin 11d-UTP (Roche) using a random primer reaction.

\section{In situ hybridisation}

Polytene chromosome squashes were prepared from $D$. buzzatii salivary glands of third-instar larvae as described by Labrador et al. [56]. Prehybridisation, hybridisation and post-hybridisation washes were performed following a protocol by Roche [57].

In situ hybridisation is a reliable technique and the most suitable method for detecting and locating insertions in chromosomal arms. However, the power of resolution of this technique is limited in cases of discrimination of very close sites on the chromosome or elements whose sequence has a divergence below $10 \%$. In the present study, where only a few sites were detected, this is not a drawback.

\section{Inversions}

Inversions were identified after microscopic observation of the same slides used for in situ hybridisation. Inversion frequencies were calculated by dividing the number of inversions of each type by the total number of individuals analysed.

\section{Statistical analyses}

Most statistical analyses and graphs were performed using the statistical software SPSS version 15.0 and Excel. In cases of multiple testing, Bonferroni's correction was applied to the data [58].

\section{Acknowledgements}

We acknowledge the invaluable assistance provided by JSF Barker in the planning of and the helping in the field work. The field assistance by H. Ruíz 
and the technical laboratory assistance by M. Peiró are also acknowledged. Two anonymous reviewers contributed with their helpful discussions and comments to improve the manuscript. The field work was carried out during a sabbatical stay of AF, sponsored by a research grant from Generalitat de Catalunya, in Ary Hoffman's laboratory at the University of Melbourne (Australia) for which we are highly indebted to him. BioMedES Ltd assisted us to improve the English of this manuscript. This research was supported by grants BOS2003-05904-C02-01 and CGL2006-13423-C02-01/02 from the Ministerio de Educación y Ciencia (Spain), grants CGL2009-12912-C03-01 and CGL2010-15395 from the Ministerio de Ciencia e Innovación (Spain), and grants 2005SGR 00995 and 2009SGR 636 from Generalitat de Catalunya to the Grup de Biologia Evolutiva.

\section{Authors' contributions}

MPGG participated in the design, the chromosomal slides, in situ hybridisation, statistical analyses and the writing of the manuscript. AF participated in the design, collected Australian populations, directed the project and contributed to writing the manuscript. Both authors read and approved the final manuscript

Received: 26 July 2010 Accepted: 24 April 2011 Published: 24 April 2011

\section{References}

1. Biémont C, Vieira C: Junk DNA as evolutionary force. Nature 2006, 443:521-524

2. Montgomery E, Huang S, Langley C, Judd B: Chromosome rearrangement by ectopic recombination in Drosophila melanogaster-Gene structure and evolution. Genetics 1991, 129:1085-1098.

3. Montgomery EA, Charlesworth B, Langley C: A test for the role of a natural selection in the stabilization of transposable element copy number in a population of Drosophila melanogaster. Genet Res 1987, 49:31-41.

4. Tsitrone : Dynamics of transposable elements under the selection model. Genet Res 1999, 74:159-164.

5. Decelière $\mathrm{G}$, Charles $\mathrm{S}$, Biémont $\mathrm{C}$ : The dynamics of transposable elements in structured populations. Genetics 2005, 169:467-474.

6. Lockton S, Gaut B: The evolution of transposable elements in natural populations of self-fertilizing Arabidopsis thaliana and its outcrossing relative Arabidopsis lyrata. BMC Evol Biol 2010, 10:10, 10 2010, 10:10.

7. Dolgin E, Charlesworth B, Cutter A: Population frequencies of transposable elements in selfing and outcrossing Caenorhabditis nematodes. Genet Res 2008, 90(4):317-329.

8. Barker J: Population genetics of Opuntia breeding Drosophila in Australia. In Ecological Genetics and Evolution: The cactus-yeast-Drosophila model system. Edited by: Barker JS, WT. Sydney, Australia: Academic Press; 1982:209-224.

9. Sokal R, Oden N, Barker J: Spatial structure in Drosophila buzzatii populations: simple and directional spatial autocorrelation. Am Nat 1987, 129:122-142

10. Barker J, Frydenberg J, González J, Davies H, Ruiz a, Sorensen J, Loeschcke : Bottlenecks, population differentiation and apparent selection at microsatellite loci in Australian Drosophila buzzatii. Heredity 2009, 102:389-401.

11. Pantazidis A, Labrador M, Fontdevila A: The retrotransposon Osvaldo from Drosophila buzzatii displays all structural features of a funcrional retrovirus. Mol Biol Evol 1999, 16(7):909-921.

12. García Guerreiro M, Fontdevila A: Molecular characterization and genomic distribution of Isis: a new retrotransposon of Drosophila buzzatii. Mol Genet Genomics 2007, 277:83-95.

13. Sokal R, Rohlf F: Biometry. New York: Freeman; 31995

14. Fisher RA: Statistical methods for research workers. New York: Hafner, 13 1958.

15. Wharton L: Analysis of the repleta group of Drosophila. Univ Tex Publ 1942, 4228:23-53.

16. García Guerreiro MP, Fontdevila A: Chromosomal distribution of the transposable elements Osvaldo and blanco in original and colonizer populations of Drosophila buzzatii. Genet Res 2001, 77:227-238.

17. Labrador M, Seleme MDC, Fontdevila A: The evolutionary history of Drosophila buzzatii. XXXIV. The distribution of the retrotransposon Osvaldo in original and colonizing populations. Mol Biol Evol 1998, 15:1532-1547.
18. Ruíz A, Naveira H, Fontdevila A: La historia evolutiva de D. buzzatii. IV. Aspectos citogenéticos de su polimorfismo cromosómico. Genét lbér 1984, 36:13-35.

19. Fontdevila A: Colonizing Species of Drosophila. In Molecular Techniques in Taxonomy. Volume 57. Edited by: Hewitt GM, Johnston A, Young J. Heidelberg: Springer-Verlag; 1991:249-269.

20. Knibb W, Barker J: Polymorphic inversion and esterase loci complex on chromosome 2 of Drosophila buzzatii II. Spatial variation. Aust J Biol Sci $1988,40: 257-269$.

21. Bartolomé C, Maside $X$, Charlesworth B: On the abundance and distribution of transposable elements in the genome of Drosophila melanogaster. Mol Biol Evol 2002, 19:926-937.

22. Rizzon C, Marais G, Gouy M, Biémont C: Recombination rate and the distribution of transposable elements in the Drosophila melanogaster genome. Genome Res 2002, 12:400-407.

23. Langley $\mathrm{CH}$, Montgomery EA, Hudson R, Kaplan N, Charlesworth B: On the role of unequal exchange in the containment of transposable element copy number. Genet Res 1988, 52:223-235.

24. García Guerreiro MP, Fontdevila A: The evolutionary history of Drosophila buzzatii. XXXVI. Molecular structural analysis of Osvaldo retrotransposon insertions in colonizing populations unveils drift effects in founder events. Genetics 2007, 175:301-310.

25. García Guerreiro M, Chávez-Sandoval B, Balanyà J, Serra L, Fontdevila A: Distribution of the transposable elements bilbo and gypsy in original and colonizing populations of Drosophila subobscura. BMC Evolutionary Biology 2008, 8:234.

26. Freeman D: Prickly pear menace in eastern Australia. Geographical Review 1992, 82(4):413-429.

27. Barker J, Krebs RA, Davies HI: Geographical distributions, relative abundance and coexistence of Drosophila aldrichi and Drosophila buzzatii in Australia. Austral Ecology 2005, 30:546-557.

28. Barker J, Mulley J: Isozyme variation in natural populations of Drosophila buzzatii. Evolution 1976, 30:213-233.

29. Frydenberg J, Pertoldi C, Dahlgaard J, Loeschcke V: Genetic variation in original and colonizing Drosophila buzzatii populations analysed by microsatellite loci isolated with a new PCR screening method. Mol Ecol 2002, 11:181-190.

30. Halliburton R, Barker J: Lack of mitochondrial DNA variation in Australian Drosophila buzzatii. Mol Biol Evol 1993, 10(2):484-487.

31. Barker J, Sene F, East P, Pereira M: Allozyme and chromosomal polymorphism of Drosophila buzzatii in Brazil and Argentina. Genetica 1985, 67:161-170.

32. DeYoung, Honeycutt R: The molecular toolbox: genetic techniques in wildlife ecology and management. J Wild Manag 2005, 69:1362-1384.

33. Hawley D, Briggs J, Dhondt A, Lovette I: Reconciling molecular signatures across markers: mitochondrial DNA confirms founder effect in invasive North American house finches (Carpodacus mexicanus). Conserv Genet 2008, 9:637-643.

34. Wright S: Evoution and the genetics of populations I. Univ. Chicago; 1977III.

35. Morgan M: Transposable element number in mixed mating populations. Genet Res 2001, 77:261-275.

36. Andolfatto $P$ : Regions of lower crossing harbor more rare variants in African populations of Drosophila melanogaster. Genetics 2001, 158:657-665.

37. Begun D, Aquadro C: African and North American populations of Drosophila melanogaster are very different at the DNA level. Nature 1993, 365:548-550.

38. González J, Lenkov K, Lipatov M, Macpherson J, Petrov D: High rate of recent transposable element-induced adaptation in Drosophila melanogaster. PLOS Biology 2008, 6(10):2109-2129.

39. Vieira C, Lepetit D, Dumont S, Biémont C: Wake Up of Transposable Elements Following Drosophila simulans Worldwide Colonization. $\mathrm{Mo}$ Biol Evol 1999, 16(9):1251-1255.

40. Grandbastien M: Activation of plant retrotransposons under stress conditions. Trends in plants science 1998, 3(5):181-187.

41. Labrador M, Fontdevila A: High transposition rates of Osvaldo, a new Drosophila buzzatii retrotransposon. Mol Gen Genet 1994, 245:661-674.

42. Parsons P, Bock I: The population biology of Australian Drosophila. Ann Rev Ecol Syst 1979, 10:229-245. 
43. Mann J: Cacti naturalised in Australia and their control. SG Reid Government 1970.

44. Biémont C, Aouar A, Arnault C: Genome reshuffling of the copia element in an inbred line of Drosophila melanogaster. Nature 1987, 329:742-744.

45. Yang H, Hung T, You T, Yang T: Genomewide Comparative Analysis of the Highly Abundant Transposable Element DINE-1 Suggests a Recent Transpositional Burst in Drosophila yakuba. Genetics 2006, 73(1):189-196.

46. Naito K, Zhang F, Tsukiyama T, Saito H, Hancock C, Richardson A, Okumoto $Y$, Tanisaka T, Wessler S: Unexpected consequences of a sudden and massive transposon amplification on rice gene expression. Nature 2009, 46:1130-1134.

47. Salzberga A, Fisherb O, R ST, Ankrib S: Identification of methylated sequences in genomic DNA of adult Drosophila melanogaster. Biochemical and Biophysical Research Communications 2004, 322:465-469.

48. Rebollo R, Lerat E, Lopez Kleine L, Biémont C, Vieira C: Losing helena: The extinction of a drosophila LINE-like element. BMC genomics 2008, 9:149.

49. Brennecke J, Aravin A, S A, D M, K M, S R, H GJ: Discrete small RNAgenerating loci as master regulators of transposon activity in Drosophila. Cell 2007, 128:1089-1103.

50. Charlesworth B, Sniegowsky P, Stephan W: The evolutionary dynamics of repetitive DNA in eukaryotes. Nature 1994, 371:215-220.

51. Goldman ASH, Lichten M: The efficiency of meiotic recombination between dispersed sequences in Saccharomyces cerevisiae depends upon their chromosomal location. Genetics 1996, 144:43-55.

52. Charlesworth B, Lapid A, Canada D: The distribution of transposable elements within and between chromosomes in a population of Drosophila melanogaster. I. Element frequencies and distribution. Genet Res 1992, 60:103-114.

53. Charlesworth B, Lapid A, Canada D: The distribution of transposable elements within and between chromosomes in a population of Drosophila melanogaster. II. Inferences on the nature of selection against elements. Genet Res 1992, 60:115-130.

54. Sniegowsky PD, Charlesworth B: Transposable element numbers in cosmopolitan inversions from a natural population of Drosophila melanogaster. Genetics 1994, 137:815-827.

55. Charlesworth B, Charlesworth D: Transposable elements in inbreeding and outbreeding populations. Genetics 1995, 140:415-417.

56. Labrador M, Naveira H, Fontdevila A: Genetic mapping of the Adh locus in the repleta group of Drosophila by in situ hybridization. J Hered 1990, 81:83-86.

57. Smidt E: A simplified and efficient protocol for non-radioactive in situ hybridization to polytene chromosomnes with a DIG-labeled DNA probe. In Non-radioactive In situ hybridization Aplication Manual. Edited by: Grünewald-Janho S, Keesey J, Leous M, van Miltenburg R, Schroeder C. Mannheim: Roche; 1996:97-107.

58. Holm S: A simple sequencially rejective multiple test procedure. Scand J Stat 1979, 6:65-70.

doi:10.1186/1471-2148-11-111

Cite this article as: García Guerreiro and Fontdevila: Osvaldo and Isis retrotransposons as markers of the Drosophila buzzatii colonisation in Australia. BMC Evolutionary Biology 2011 11:111.

\section{Submit your next manuscript to BioMed Central and take full advantage of:}

- Convenient online submission

- Thorough peer review

- No space constraints or color figure charges

- Immediate publication on acceptance

- Inclusion in PubMed, CAS, Scopus and Google Scholar

- Research which is freely available for redistribution 